\title{
PRODUÇÃO E QUALIDADE DE MEL NA ZONA DA MATA DE PERNAMBUCO
}

\author{
Renata Valéria Regis de Sousa Gomes ${ }^{1}$; Maria Elaine de Miranda ${ }^{1}$; Escarião da \\ Nóbrega Gomes ${ }^{2}$; Daiana da Silva Sombra ${ }^{3}$; Jean Berg Alves da Silva ${ }^{3}$ \\ 'Departamento de Zootecnia, Universidade Federal Rural de Pernambuco, Recife, \\ PE, Brasil; (renatav_sousa@hotmail.com) \\ ${ }^{2}$ Pós-graduação em Morfotecnologia, Universidade Federal de Pernambuco, Recife, \\ PE, Brasil \\ ${ }^{3}$ Pós-graduação em Ciência Animal, Universidade Federal Rural do Semi-árido, \\ Mossoró, RN, Brasil
}

Recebido em: 02/10/2017 - Aprovado em: 21/11/2017 - Publicado em: 05/12/2017 DOI: 10.18677/EnciBio_2017B52

\begin{abstract}
RESUMO
A atividade apícola tem se consolidado como boa alternativa para geração de emprego e renda, apresentando o mel da região Nordeste do Brasil uma grande aceitação no mercado, pelo seu diferencial do mel ser proveniente de vegetação nativa. O presente trabalho teve como objetivo descrever a atividade apícola na região da zona da mata sul de Pernambuco, com ênfase na produção e qualidade dos méis produzidos nessa região. Para quantificar a produção de mel foram utilizadas as informações do banco de dados da Associação de Apicultores e Meliponicultores do Cabo. Para análise da qualidade do mel, foram coletadas amostras e submetidas às análises sensorial dos méis quanto a cor e análises físicoquímicas de umidade, acidez, pH, Hidroximetilfurfural (HMF) e atividade de água (Aw). Todas as análises foram realizadas em duplicata e todos os dados analisados descritivamente. A apicultura na região da zona da mata de Pernambuco é uma atividade predominantemente familiar, que gera renda complementar para a família, e que apesar da aptidão da região para produção de mel, a produção está subestimada. Todas as amostras de méis analisadas dos apiários de produção apresentaram qualidade e estavam de acordo com o estabelecido pela legislação brasileira.
\end{abstract}

PALAVRAS-CHAVE: abelha, africanizada, apicultura, Apis mellifera.

\section{PRODUCTION AND HONEY QUALITY IN PERNAMBUCO ZONA DA MATA}

\begin{abstract}
Beekeeping activity has consolidated itself as a good alternative for employment and income generation, presenting Brazil northeastern region honey with a great acceptance in the market, because of its differential of honey, which comes from native vegetation. This work had aimed to describe beekeeping activity in Pernambuco South Zona da Mata region, emphasizing honey production and quality of this region. In order to quantify the production of honey, information from database ENCICLOPÉDIA BIOSFERA, Centro Científico Conhecer - Goiânia, v.14 n.26; p. 5392017
\end{abstract}


of the Association of Beekeepers and Meliponicultores of Cabo was used. For honey quality analysis, samples were collected and submitted to honey sensorial analysis for color and moisture physical-chemical analyzes, acidity, $\mathrm{pH}$, hydroxymethylfurfural (HMF) and water activity (WA). All analyzes were performed in duplicate and all data were descriptively analyzed. Beekeeping in Pernambuco Zona da Mata is a predominantly family activity, which generates supplementary income for the family, and despite the region's ability to produce honey, production is underestimated. All honey samples analyzed from the apiary of production presented a good quality and they were in agreement with the established by the Brazilian legislation.

KEYWORDS: bee, africanized, beekeeping, Apis mellifera.

\section{INTRODUÇÃO}

O Nordeste brasileiro que até poucos anos não era conhecido como produtor de mel, hoje é considerado como região de alta produção apícola, com aproximadamente $33 \%$ de participação na produção nacional, sendo, a apicultura nordestina uma atividade que tem se consolidado como boa alternativa de geração de emprego e renda, e uma das atividades que mais promove a inclusão social do homem do campo, especialmente o pequeno produtor (GONÇALVES et al., 2010).

No Estado de Pernambuco, a apicultura se institucionalizou em 1967, com a criação da Cooperativa dos Apicultores de Pernambuco (CAPEL), primeira Cooperativa apícola do Brasil (ARAÚJO, 2006). Após a criação da CAPEL, as primeiras políticas para o Estado vieram em 1977, com o Projeto Sertanejo/SUDENE, que foi o primeiro programa voltado para a apicultura do Governo Federal e introduziu a cultura apícola na região e, em 1988, veio o Programa de Expansão da Atividade Apícola no Estado - PROMEL (LEÃO et al., 2012).

As características de clima e flora dessa região a coloca competitivamente no mercado de produtos apícolas, exercendo o clima e a flora uma grande influência na produção de mel (VIDAL, 2017), sendo que as variações climáticas influenciam fortemente sobre a dinâmica da vegetação de uma região (GALVINCIO et al., 2016). No ano de 2011, Pernambuco apresentava três (03) municípios entre os 20 mais produtores de mel do país, com participação na produção nacional de 5,5\%, sendo que em 2012, a produção de mel foi fortemente afetada pela escassez de chuvas nas principais regiões produtoras (IBGE, 2015).

O Estado é caracterizado por dois climas, tropical úmido (predominante no litoral) e semiárido (predominante no interior) (APAC, 2015), sendo o período mais crítico para a atividade apícola nas regiões de clima semiárido a época de seca, em que na maioria das regiões produtoras, por não contarem mais com a diversidade da vegetação nativa, há necessidade de alimentar os enxames e proteger as colmeias das altas temperaturas (VIDAL, 2014). Fator inverso ocorre na região da zona da mata de Pernambuco, que localizada numa faixa litorânea, possui na sua vegetação resquício de mata atlântica, áreas de manguezal e zona canavieira, sendo no período seco a época de floração nessa região, e a época crítica para a atividade apícola, o período das chuvas contínuas.

Considerando a importância econômica, social e ambiental da apicultura, e a necessidade de ações com vistas ao desenvolvimento e sustentabilidade da atividade apícola na zona da mata, o objetivo dessa pesquisa foi descrever o perfil da atividade apícola na região da zona da mata sul de Pernambuco com ênfase na produção e qualidade dos méis produzidos nessa região. 


\section{MATERIAL E METÓDOS}

A região da zona da mata de Pernambuco possui em sua composição original cobertura vegetal correspondente à Mata Atlântica, ocorrendo variações sensíveis da vegetação, que a divide em subzonas com áreas de manguezal (LIMA, 2007). Sua vegetação exuberante e diversidade biológica têm sido, desde os tempos coloniais, destruídas pelo ciclo da cana-de-açúcar (Saccharum spp.) e do pau-brasil (Paubrasilia echinata Lam. Gagnon, H.C.Lima \& G.P.Lewis), originando nessa região áreas de zona canavieira, e nos dias atuais, o desmatamento vem sendo realizado para a implantação de indústrias (SILVA, 2017), o que vem acentuando o processo de fragmentação dos remanescentes de Mata Atlântica dessa microrregião (PEREIRA et al., 2013).Para quantificar a produção de mel foram utilizadas as informações do banco de dados da Associação de Apicultores e Meliponicultores do Cabo (AAMC), que fica localizada no município do Cabo de Santo Agostinho, região do litoral e zona da mata sul de Pernambuco, Brasil. A AAMC é formada por trinta e três associados e foram utilizados os dados (número de colmeias, quantidade de colheitas e a média de produção anual) registrados na AAMC no período de março de 2015 a março de 2016.

Para análise da qualidade do mel, foram coletadas sete amostras de méis de abelhas africanizadas (Apis mellifera L.), de quatro apiários dos associados da AAMC, escolhidos aleatoriamente e localizados em região com vegetação de resquício de mata atlântica, área de manguezal, área canavieira e vizinho a uma fábrica de refinação de milho. A primeira amostra de mel de cada apiário foi da colheita realizada no mês de dezembro de 2015 e a segunda amostra da colheita realizada no mês de janeiro de 2016. Do apiário localizado vizinho a uma fábrica de refinação de milho só se obteve amostra da colheita do mês de dezembro de 2015. Os méis foram recebidos em frascos de polietileno estéreis de $500 \mathrm{~mL}$, identificados com um número de registro em uma ficha contendo informação sobre o local de produção, data da centrifugação, recebimento e identificação da origem. As amostras foram submetidas às análises sensorial e físico-químicas.

Para a análise sensorial do mel quanto a cor, foi utilizado o espectrofotômetro (METROLAB 1700 UV VIS), com medição em 635 nm, solução a 50\% usando a glicerina como o branco (ESCOBAR-MARTINEZ et al., 1992), com as leituras obtidas em colorímetro de Pfund. Nas análises físico-químicas, a determinação do $\mathrm{pH}$ foi baseada em titulação simples, utilizando pHmetro Digimed DMPH-1 (MORAES; TEIXEIRA, 1998). A atividade de água (Aw) foi determinada pelo ponto de orvalho utilizando o equipamento AquaLab 3TE (DECAGON, 2005).

Para verificar a acidez, as amostras foram pesadas e diluídas e adicionado três gotas de fenoftaleína, em seguida realizada a titulação com solução de hidróxido de sódio $(\mathrm{NaOH} 0,1 \mathrm{~N})$, obtendo-se o valor da acidez multiplicando o valor do titulado na bureta, vezes o peso da amostra (MARCHINI et al., 2005).

Para determinação do Hidroximetilfurfural (HMF) o método utilizado foi 0 quantitativo, através da espectofotometria a 284 e $336 \mathrm{~nm}$ (BOGDANOV et al., 1997). A análise da umidade foi realizada utilizando-se o refratômetro Atago, com correção de temperatura automática, que mede de 12 a $30 \%$ de umidade, em que foram colocadas duas gotas do mel e em seguida fizeram-se as leituras. Todas as análises foram realizadas em duplicata, no Laboratório de Inspeção de Produtos de Origem Animal (LIPOA), do Departamento de Ciência Animal da Universidade 
Federal Rural do Semi-árido, Mossoró, RN, Brasil. Todos os dados foram tabulados e analisados descritivamente.

\section{RESULTADOS E DISCUSSÃO}

Analisando-se os registros do banco de dados da Associação de Apicultores e Meliponicultores do Cabo (AAMC), observou-se que o mel é o único produto apícola explorado e que, $57,6 \%$ dos apiários possuem de duas a seis colmeias; $36,4 \%$ possuem de 10 a 35 colmeias; e dois apiários que representam 6\%, possuem 75 e 112 colmeias (Figura 1), contabilizando-se um total de 468 colmeias, com média de $14,18 \pm 22,43$ colônias distribuídas nos 33 apiários registrados na AAMC. Oliveira et al. (2016) observaram que na região Nordeste do Brasil vem crescendo o número de cooperativas voltadas para a apicultura e meliponicultura, sendo necessário capacitação, crédito, assistência técnica e extensão rural, para que se amplie as vias de acesso ao crescimento, o que desenvolverá o processo de geração e complementariedade de renda familiar, como verificado no Rio Grande do Norte por Pereira et al. (2015), que mais de $58 \%$ dos agricultores utilizam a apicultura como fonte de renda, apresentando a atividade apícola grande importância econômica, sendo a apicultura nordestina uma atividade de caráter eminentemente familiar, onde existem cerca de 46.356 apicultores em toda a Região e a maioria possui até 200 colmeias (VIDAL, 2013).

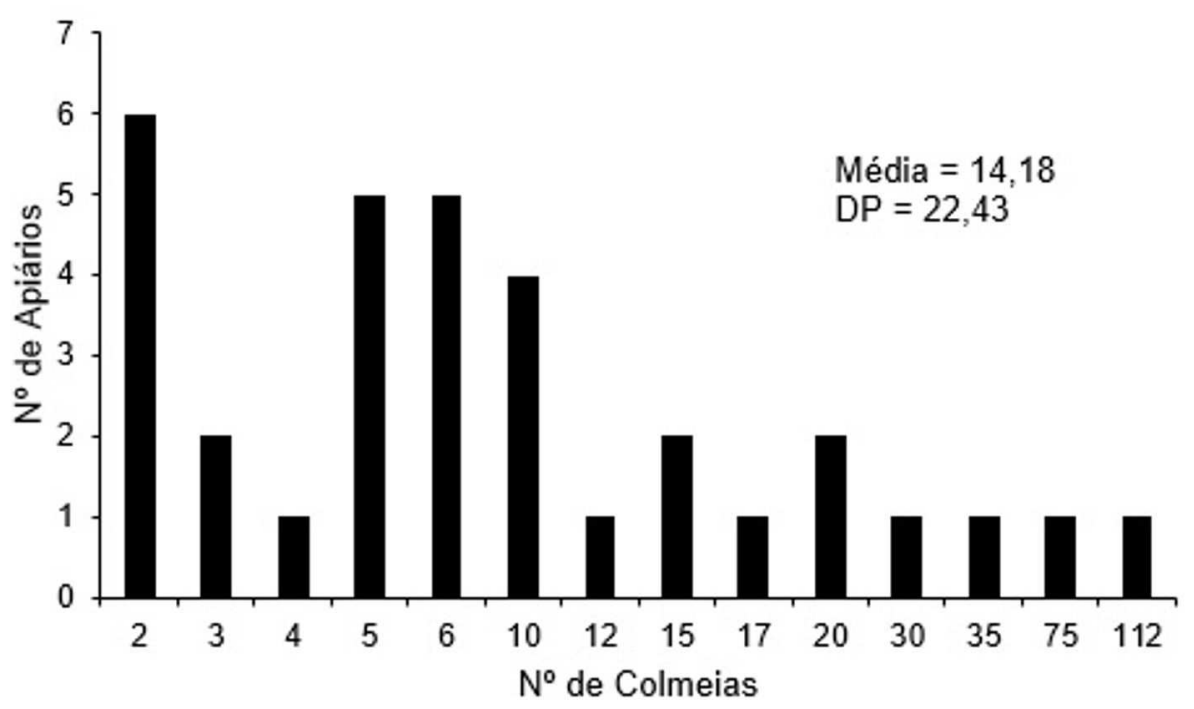

FIGURA 1. Média, desvio padrão (DP) e frequência do número de colmeias distribuídas em 33 apiários registrados na Associação de Apicultores de Meliponicultores do Cabo (AAMC).

De acordo com os registros da AAMC, a produção média de mel beneficiado na associação, no período de março de 2015 a março de 2016 , foi de $13.890 \mathrm{Kg}$ de mel, quantitativo obtido em 5 colheitas, com média anual de aproximadamente $30 \mathrm{Kg}$ de mel/colmeia/ano. A comercialização desse mel foi feita de duas formas: venda direta ao consumidor ou através da AAMC, que por meio de licitação vendeu o mel para a prefeitura/Estado para ser distribuído na merenda escolar. Na venda direta ao consumidor, o mel foi vendido a $R \$ 30,00$ a garrafa com $1 / \mathrm{Kg}$ e através da associação, o valor vendido foi de $\mathrm{R} \$ 14,00 / \mathrm{Kg}$ de mel. 
A forma de apresentação do produto influencia no preço de venda, como observado por Silva et al. (2017) na Associação dos Apicultores do Município de São Rafael -RN, onde o mel em bisnaga de $500 \mathrm{~g}$ obtinha o preço de venda de $\mathrm{R} \$$ $24,00 / \mathrm{Kg}$, já nos casos que não ocorria o beneficiamento do mel, o preço caia para em torno de $\mathrm{R} \$ 8,00 / \mathrm{Kg}$ do mel bruto. De acordo com Gonçalves et al. (2014) é importante para o sucesso da atividade apícola, em que prevalece o caráter familiar, uma mudança organizacional acompanhada da profissionalização da atividade, o que acarretará na formação dos sócios-proprietários, tornando o conhecimento mais especializado e técnico. Como é o caso da importância do conhecimento da vegetação das áreas no entorno do apiário para manutenção da atividade apícola, por a escassez de floração proporcionar baixa produção, causando perda de renda líquida para os apicultores (CASTILHOS et al., 2016). E o conhecimento das vantagens para os produtores da troca de rainhas, o que é uma prática pouco estabelecida pelo apicultor devido, em parte, ao tipo de manejo que é realizado nos apiários de produção e, em parte, ao desconhecimento das vantagens (MARTINEZ; SOARES 2012).

Nas análises das características organolépticas das amostras de méis, observou - se variação da coloração dos méis das regiões estudadas, tanto entre apiários, como no mesmo apiário em data diferente da colheita, ao que se atribui aos diferentes períodos de floração das espécies florais da região, sendo que todas as amostras analisadas estavam dentro dos padrões do MAPA (2000), que estabelece que o mel deve apresentar características organolépticas tais, como a cor levemente amarelada à castanho escuro.

Verificou-se na zona canavieira, que a coloração do mel variou entre âmbar, âmbar claro e âmbar escuro (Tabela 1). A colheita de mel escuro coincidiu com a época de corte da cana de açúcar, que ocorre do mês de setembro a dezembro. Esse mel não é de flores, mas elaborado a partir da seiva da cana dos canaviais cortados, tem valor nutritivo superior ao melado, é muito escuro, pouco viscoso, com forte aroma de rapadura. Já o mel claro, que é produzido após o período de corte da cana, deve-se ao resquício de mata atlântica que ainda existe nessa região.

TABELA 1. Características organolépticas (Cor) e físico-químicas ( $\mathrm{pH}$; atividade de água (Aw); acidez; Hidroximetilfurfural (HMF); e umidade) dos méis coletados em dezembro/2015 e janeiro/2016 de quatro apiários localizados em áreas canavieira, mangue, resquício de mata atlântica e nas mediações de uma indústria de refinação de milho na região do litoral e zona da mata sul de Pernambuco.

\begin{tabular}{c|c|c|c|c|c|c|c}
\hline $\begin{array}{c}\text { Amostras/ } \\
\text { Área }\end{array}$ & $\begin{array}{c}\text { Mês/Ano } \\
\text { da Coleta }\end{array}$ & Cor & $\mathbf{p H}$ & Aw & $\begin{array}{c}\text { Acidez } \\
\mathbf{m E q} / \mathbf{K g}\end{array}$ & $\begin{array}{c}\text { HMF } \\
\mathbf{M g} / \mathbf{g}\end{array}$ & Umidade\% \\
\hline Canavieira & Dez/2015 & Âmbar escura & 4,10 & 0,6520 & 44,8 & 5,38 & 18,1 \\
& Jan/2016 & Âmbar claro & 4,27 & & 23 & 1,06 & 19,6 \\
Mangue & Dez/2015 & Âmbar & 4,01 & 0,6620 & 34,3 & 0,38 & 19,3 \\
& Jan/2016 & Âmbar claro & 4,28 & & 22,3 & 1,34 & 19,7 \\
$\begin{array}{c}\text { Resquício de } \\
\text { Mata atlântica }\end{array}$ & Dez/2015 & Âmbar claro & 4,09 & 0,6307 & 33,5 & 2,69 & 17,9 \\
$\begin{array}{c}\text { Refinação de } \\
\text { milho }\end{array}$ & Jan/2016 & Âmbar claro & 4,09 & & 33,5 & 2,69 & 17,9 \\
\hline
\end{tabular}


$\mathrm{Na}$ área de manguezal, observou-se que as colorações dos méis variaram de âmbar a âmbar claro. Nas amostras da zona da mata não ocorreram variações na coloração do mel nas duas datas coletadas. E o mel do apiário localizado perto da refinação de milho, apresentou coloração classificada como Âmbar claro (Tabela 1).

Mantilla et al. (2012) relataram que propriedades sensoriais do mel, tais como a cor, variam de acordo com as condições geográficas e sazonais, bem como a origem floral. As grandes variedades de méis de abelha podem ser diferenciadas pela origem floral, onde o mel procedente do néctar das flores de uma única espécie vegetal é classificado como monoflorais, ou de várias espécies vegetais classificado como méis poliflorais (ARNAUD et al., 2008).

As análises de méis são necessárias para dar certeza ao consumidor da qualidade do produto comprado e respaldar o apicultor quanto a excelência do produto por ele produzido e comercializado sendo que, as características físicoquímicas dos méis dependem de muitos fatores como, espécies de abelha, aspectos climáticos, estado fisiológico da colmeia e espécies vegetais de onde é coletado o néctar e pólen (DAMASIA-GOMES et al., 2015).

Nas análises físico-químicas, os valores de $\mathrm{pH}$ obtidos nas amostras variaram de 3,87 a 4,28 (Tabela 1), estando de acordo com o limite estabelecido pela Normativa $n^{\circ} 11$ do Ministério da Agricultura Pecuária, que é de 3,3 a 4,6. Evangelista-Rodrigues et al. (2005) avaliando diferentes tipos de méis de Apis melífera das regiões do Cariri e Brejo paraibano, verificaram pH de 3,85 e 4,61 demostrando diferenças significativas.

$\mathrm{Na}$ atividade de água $(\mathrm{Aw})$ foi possível observar que os valores variaram de 0,604 a 0,662 (Tabela 1) estando todas as amostras de acordo com os padrões dos méis brasileiros de Apis que é entre 0,54 a 0,75 (BRASIL, 2000). Kuroishi et al. (2012) observaram que amostras de méis armazenadas a uma temperatura de $31^{\circ} \mathrm{C}$, com o passar do tempo há uma tendência de aumento da atividade de água, e que em temperaturas menores $\left(21^{\circ} \mathrm{C}\right.$ e $\left.11^{\circ} \mathrm{C}\right)$, o aumento da atividade de água favorece a cristalização do mel.

Levando-se em consideração que é possível ocorrer alteração no mel decorrente das condições climáticas, da florada presente na região, do período de desenvolvimento do vegetal, da espécie da abelha, além do processamento e armazenamento do mel (GARSKE; BUDEL, 2012), a cristalização é um fator a ser considerado quando o mel é envasado, distribuído e comercializado, por a maioria dos méis cristalizar facilmente, por ser um líquido muito saturado de açúcares, em que o tamanho dos cristais formados tem relação com as funções glicose/água, frutose/glicose e histórico térmico, apresentando-se como principal desvantagem da cristalização, a dificuldade de servir o mel após o produto ser envasado (GOIS et al., 2013).

A acidez das amostras de méis analisadas, variaram de 22,3 a $44,8 \mathrm{mEq} / \mathrm{kg}$ (Tabela 1), estando todas as amostras dentro dos padrões exigidos pela legislação brasileira, que recomenda o limite máximo de $50 \mathrm{mEq} / \mathrm{kg}$ de acidez no mel (BRASIL, 2000). Nas regiões do Cariri e Vale do Jaguaribe no Ceará, Oliveira e Santos (2011) observaram teores de acidez nos méis de abelha africanizada variando de 20,23 a $118,14 \mathrm{mEq} / \mathrm{kg}$, atribuindo essa variação aos ácidos orgânicos das diferentes fontes de néctar, pela ação da enzima glicose-oxidase que origina o ácido glucônico e, pela quantidade de minerais presentes no mel.

Analisando os resultados quanto ao constituinte hidroximetilfurfural (HMF), observou-se que o mel do apiário próximo a refinação de milho, obteve o valor 
máximo $6,34 \mathrm{mg} / \mathrm{kg}$ (Tabela 1), o que pode ser justificado pelo fato das abelhas coletarem resíduos da fábrica como alimento e o xarope de milho apresentar altos teores de HMF (GARSKE; BUDEL, 2012). Já o mel da área de mangue obteve o menor valor $0,38 \mathrm{mg} / \mathrm{kg}$ (Tabela 1 ). O HMF no mel resulta da decomposição da frutose, apresentando em baixa quantidade ou inexistente no mel fresco, sendo utilizado como um indicador de sobreaquecimento do mel ou envelhecimento durante o armazenamento, o que indica a deterioração da qualidade do produto (KOWALSKI, 2013). Todas as amostras analisadas estavam em conformidade com - Regulamento Técnico de Identidade e Qualidade do Mel do Ministério da Agricultura e do Abastecimento (BRASIL, 2000) que estabelece o máximo de 60 $\mathrm{mg} / \mathrm{Kg}$.

Quanto as análises da umidade dos méis, os resultados apresentaram valores que variaram de $16,4 \%$ a $19,7 \%$ estando todas as amostras dentro do padrão preconizado pela legislação (Tabela 1), a amostra que apresentou o menor percentual de umidade foi o mel da refinação de milho com $16,4 \%$ e o com maior percentual foi o da área de mangue com 19,7\%. De acordo com a normativa brasileira, a umidade do mel não pode ultrapassar valores acima de $21 \%$, pois se ultrapassar esse valor, o mel não está apto para a comercialização. O que reforça a importância do apicultor produzir um produto com qualidade, levando-se em consideração também que o comércio apícola vem ganhando cada vez mais espaço no agronegócio brasileiro, o que promove a fixação do homem no campo com qualidade de vida e geração de renda, como observado no Estado de Goiás que no período de 16 anos (1998 a 2013) teve um aumento de $308 \%$ na produção de mel, gerando cifras na casa dos $R \$ 21$ milhões, colocando a apicultura como uma alternativa para a diversificação de renda e/ou como atividade principal nas propriedades rural (ARAÚJO et al., 2015).

\section{CONCLUSÃO}

Concluímos que a apicultura na região da zona da mata de Pernambuco é uma atividade predominantemente familiar, que gera renda complementar para a família, e que apesar da aptidão da região para produção de mel, a produção está subestimada.

Todas as amostras de méis analisadas dos apiários de produção localizados nas áreas de mangue, mata atlântica, canavieira e vizinho a uma fábrica de refinação de milho da zona da mata sul de Pernambuco, apresentaram qualidade e estavam de acordo com o estabelecido pela legislação brasileira.

\section{REFERÊNCIAS}

APAC - Agência Pernambucana de águas e clima. Disponível em: < http://www.apac.pe.gov.br/meteorologia/estacoes-do-ano.php?> Acesso em 26 mai. 2015.

ARAÚJO, F. D.; SILVA-NETO, C. M.; RIBEIRO, A. C. C.; OlIVEIRA, G. M.; NASCIMENTO, A. R. Honey economic valuation in the state of Goiás: conservation and income. Agrarian Academy, v. 2, n. 4, p. 32-40, 2015. Disponível em: < http://www.conhecer.org.br/Agrarian\%20Academy/2015b/honey.pdf>. Acesso em: 12 out. 2017. 
ARAÚJO, T. P. (Org.). Demanda por microcrédito em três arranjos produtivos de Pernambuco: Apicultura, bacia leiteira e caprinocultura. Recife: Fundação Joaquim Nabuco: Massangana, 2006.

ARNAUD, A. F.; SILVA, R. A.; ARAÚJO, L. L. S.; SANTOS JÚNIOR, R. J.; OLIVEIRA JUNIOR, D. A. Perfil sensorial de méis de Apis mellifera L., 1758 (Hymenoptera, Apidae) produzidos na microrregião de Catolé do Rocha - PB. Revista Verde, v. 3, n. 4, p. 73-85, 2008. Disponível em:< http://www.gvaa.com.br/revista/index.php/RVADS/article/view/134/0>. Acesso em: 26 set. 2017.

BOGDANOV, S.; MARTIN, P.; LULLMAN, C. Harmonized methods of the European honey commission. Apidologie, p. 1-59, 1997. Disponível em:< http://www.ihcplatform.net/ihcmethods2009.pdf>. Acesso em: 17 out. 2017.

BRASIL. Ministério da Agricultura, Pecuária e Abastecimento. Defesa Animal. Legislações. Legislação por Assunto. Legislação de Produtos Apícolas e Derivados. Instrução Normativa n. 11, de 20 de outubro de 2000. Regulamento técnico de identidade e qualidade do mel. Acesso em: 22 set. 2015.

CASTILHOS, D. BERGAMO, G. C.; IMPERATRIZ-FONSECA, V. L.; BELCHIOR FILHO, V. Apiculture in Rio Grande do Norte, Brazil: a four-year follow-up survey. Latin American Journal of Business Management. v. 7, n. 1, p. 96-116, 2016. Disponível em: <http://www.lajbm.com.br/index.php/journal/article/view/331/166>. Acesso em: 28 set. 2017.

DAMASIA-GOMES, L.; FALEIRO, K. M.; SANTOS, S. O.; GUIMARÃES, L. E.; SILVA-NETO, C. M. Physical-chemical characteristics of honey on Brazil. Enciclopédia Biosfera, v. 11, n. 22, p. 670-682, 2015. Disponível em:< https://www.researchgate.net/publication/285755538_PHYSICAL-

CHEMICAL_CHARACTERISTICS_OF_HONEY_ON_BRAZIL. Acesso em: 14 out. 2017.

DECAGON. Aqualab - Water active meter. Operator's Manual. [S.I.]: Decagon Devices Inc., 2005. $112 \mathrm{p}$.

ESCOBAR-MARTINEZ, C. A.; GIMENEZMEZA, G. G.; MENDONZA, Q. M. R. Mieles de abejas de flor paraguaya: composición, tipificación y normalización. San Lorenzo: Ministerio da Agricultura y Ganaderia/Universidad Nacional de Assunción/Asociación Suiza para el Desarrollo y la Cooperación. 1992. 31 p.

EVANGELISTA-RODRIGUES, A.; SILVA, E. M. S.; BESERRA, E. M. F.; RODRIGUES, M. L. Análise físico-química dos méis das abelhas Apis mellifera e Melipona scutellaris produzidos em duas regiões no Estado da Paraíba. Ciência Rural, v. 35 n. 5, 2005. Disponível em: < http://revistas.bvsvet.org.br/crural/article/viewFile/18250/19090 >. Acesso em: 28 set. 2017.

GALVINCIO, J. D.; PEREIRA, J. A. S.; FRANÇA, L. M. A.; LINS, T. M. P. Análise da variação da vegetação dos períodos secos e chuvosos através do Savi e Albedo de superfície no município de Belo Jardim - PE. REDE-Revista Eletrônica do ENCICLOPÉDIA BIOSFERA, Centro Científico Conhecer - Goiânia, v.14 n.26; p. 5462017 
PRODEMA, v. 10, n. 2, p.133-146, 2016. Disponível em: <http://www.revistarede.ufc.br/revista/index.php/rede/article/view/389/115>. Acesso em: 15 out. 2016.

GARSKE, P. A.; BUDEL, J. M. Controle da qualidade de amostras de mel de Apis mellifera L. (Hymenoptera, Apidae), procedentes da região dos Campos GeraisParaná. Cadernos da Escola de Saúde, v. 6, p.185-194, 2012. Disponível em: < http://revistas.unibrasil.com.br/cadernossaude/index.php/saude/article/view/121/120> . Acesso em: 15 out. 2017.

GOIS, G. C.; LIMA, C. A. B.; SILVA, L. T.; EVANGELISTA-RODRIGUES, A. Composição do mel de Apis mellifera: requisitos de qualidade. Acta Veterinaria Brasilica, v.7, n.2, p.137-147, 2013. Disponível em: < https://periodicos.ufersa.edu.br/index.php/acta/article/viewFile/3009/5219>. Acesso em: 14 out. 2017.

GONÇALVES, L. P.; BINOTTO, E.; CINTRA, R. F. Análise da Apicultura no Estado de Mato Grosso do Sul: um Enfoque na Mudança Organizacional. Revista de Administração IMED, v. 4, n. 2, p. 245-256, 2014. Disponível em: <https://seer.imed.edu.br/index.php/raimed/article/view/492/536>. Acesso em: 28 set. 2017.

GONÇALVES, L. S.; DE JONG, D.; GRAMACHO, K. P. A expansão da apicultura e da tecnologia apícola no Nordeste brasileiro com especial destaque para o Rio Grande do Norte. Mensagem Doce, v. 3, p. 7-15, 2010. Disponível em: < http://www.apacame.org.br/mensagemdoce/105/artigo2.htm>. Acesso em: 30 abr. 2016.

IBGE. Produção da Pecuária Municipal. Rio de Janeiro, v. 43, 2015. Disponível em:

<https://biblioteca.ibge.gov.br/visualizacao/periodicos/84/ppm_2015_v43_br.pdf.> Acesso em: 30 set. 2017.

KOWALSKI, S. Changes of antioxidant activity and formation of 5hydroxymethylfurfural in honey during thermal and microwave processing Stanisław. Food Chemistry, v. 141, p. 1378-1382. 2013. Disponível em: <https://10.1016/j.foodchem.2013.04.025> doi: 10.1016/j.foodchem.2013.04.025. Acesso em: 25 set. 2017.

KUROISHI, A. M.; QUEIROZ, M. B.; ALMEIDA, M. M.; QUAST, L. B. Avaliação da cristalização de mel utilizando parâmetros de cor e atividade de água. Brazilian Journal Food Technology, v. 15, n. 1, p. 84-91, 2012. Disponível em:< http://www.scielo.br/pdf/bjft/v15n1/09.pdf>. Acesso em: 25 set. 2017.

LEÃO, E. L. S.; MOUTINHO, L. M. G.; XAVIER, M. G. P. Condicionantes de crescimento arranjo produtivo local de apicultura na região do Araripe, Pernambuco. RACE, Unoesc, Edição Especial Agronegócios, v. 11, n. 1, p. 75 - 102, 2012. Disponível em:<http://editora.unoesc.edu.br/index.php/race/article/view/1669>. Acesso em: 24 mar. 2016. 
LIMA, D. A. Estudos fitogeográficos de Pernambuco. Anais da Academia Pernambucana de Ciência Agronômica, v. 4, p.243-274, 2007. Disponível em: < http://www.ead.codai.ufrpe.br/index.php/apca/article/view/47/44>. Acesso em: 14 out. 2017.

MANTILLA, S. P. S.; SANTOS, E. B.; BARROS, L. B.; FREITAS, M. Q. Análise descritiva quantitativa aplicada em mel de abelhas (Apis mellifera): uma revisão. Colloquium Agrariae, v. 8, n.2, p. 75-84, 2012. Disponível em:<https:// 10.5747/ca.2012.v08.n2.a081>. doi: 10.5747/ca.2012.v08.n2.a081. Acesso em: 27 set. 2017.

MAPA, Ministério da Agricultura Pecuária e do Abastecimento. Instrução normativa n.11, de 20 de outubro de 2000. Diário Oficial [da] República Federativa do Brasil, Poder Executivo, Brasília, DF, 23 out. 2000. Disponível em:<http://sistemasweb.agricultura.gov.br/sislegis/action/detalhaAto.do?method=abri rArvoreTematicaNew> Acesso em: 07 nov. 2015.

MARCHINI, L. C.; MORETI, A. C. C.; OTSUK, I. P. Análise de grupamento, com base na composição química de amostras de méis produzidos por Apis mellifera $\mathrm{L}$. no estado de São Paulo. Ciência e Tecnologia de Alimentos, v. 25, n. 1, p. 8-17, 2005. Disponível em:< http://www.redalyc.org/pdf/3959/395940073003.pdf>. Acesso em: 17 out. 2017.

MARTINEZ, O. A.; SOARES, A. E. E. Melhoramento genético na apicultura comercial para produção da própolis. Revista Brasileira Saúde Produção Animal, Salvador, v. 13, n. 4, p.982-990, 2012. Disponível em:< http://www.scielo.br/pdf/rbspa/v13n4/06.pdf>. Acesso em: 30 jan. 2016.

MORAES, R. M.; TEIXEIRA, E. W. Análise de mel. Pindamonhagaba: Instituto de Zootecnia. 1998. 41 p. Manual Técnico.

OLIVEIRA, E. N. A.; SANTOS, D. C. Análise físico-química de méis de abelhas africanizada e nativa. Revista Instituto Adolfo Lutz. v. 70, n. 2, p.132-138, 2011. Disponível em:< http://revistas.bvs-vet.org.br/rialutz/article/view/5823>. Acesso em: 28 set. 2017.

OLIVEIRA, P. A.; SÁ, M. S.; MELO, A. B.; ROCHA JUNIOR, C. J. G.; CAVALCANTE, M. C. Levantamento das organizações associativas de apicultores e meliponicultores no Brasil. Revista Econômica do Nordeste, v. 47, n. 4, p. 51-62, 2016. Disponível em: < https://ren.emnuvens.com.br/ren/article/view/617/490>. Acesso em: 27 set. 2017.

PEREIRA, D. S.; ADELINO, C. J.; OLIVEIRA, M. S.; SILVA, M.; HOLANDA-NETO, J. $P$. Apicultura como fonte de renda na comunidade de Vaca Morta no município de Marcelino Vieira - RN, Brasil. Cadernos de Agroecologia, v. 10, n. 3, 2015. Disponível em: <https://www.alice.cnptia.embrapa.br/bitstream/doc/1046251/1/19459771721PB.pdf> . Acesso em: 27 set. 2017. 
PEREIRA, R. C. A.; SILVA, J. A.; BARBOSA, J. I. S. Aspectos florísticos de uma área da mata atlântica situada na microrregião da mata meridional do Estado de Pernambuco, Brasil. Anais da Academia Pernambucana de Ciência Agronômica, v. 10, p.280-307, 2013. Disponível em: < http://www.journals.ufrpe.br/index.php/apca/article/view/317/350>. Acesso em: 12 out. 2017.

SILVA, J. M. Revisão histórica da diversidade vegetal da Zona da Mata Norte de Pernambuco com ênfase no município de goiana. Revista Espaço Acadêmico, n. 191, p. 12- 26, 2017. Disponível em: < http://ojs.uem.br/ojs/index.php/EspacoAcademico/article/view/35815/18920>. Acesso em: 15 out. 2017.

SILVA, N. V.; PAIVA, A. C. C.; MEDEIROS, A. P.; MEDEIROS, A. C.; SILVA, O. S. et al. Produção de mel no município de São Rafael: um estudo de caso com base na experiência da Associação dos Apicultores do Desterro. Revista Brasileira de Gestão Ambiental, v. 11, n.01, p.112-118, 2017. Disponível em: < http://www.gvaa.com.br/revista/index.php/RBGA/article/view/4974/4263>. Acesso em: 26 de set. 2017.

VIDAL, M. F. Efeitos da seca de 2012 sobre a apicultura nordestina. Informe Rural Etene, ano VII, n. 2, p. 1-5, 2013. Disponível em: <https://www.bnb.gov.br/documents/88765/89729/ire_ano7_n2.pdf/7a9e8843-0f574ed8-b737-0a6096c915cd>. Acesso em: 04 abr. 2016.

VIDAL, M. F. Efeitos da seca de 2012 nas exportações nordestinas de mel. Informe Rural Etene, ano VIII, n. 3, p. 1-5, 2014. Disponível em: < https://www.bnb.gov.br/documents/88765/89729/ire_ano8_n3_2014.pdf/8f2a145f4c23-4520-8ae5-6657576de434>. Acesso em: 28 set. 2017.

VIDAL, M. F. Desempenho da apicultura nordestina em anos de estiagem. Caderno Setorial Etene, ano II, n. 11, p. 1-10, 2017. Disponível em: < https://www.bnb.gov.br/documents/80223/2130269/apicultura_11_2017.pdf/6967e3c e-2381-04a5-f3d1-c00f5989a481>. Acesso em: 13 out. 2017. 